\title{
CONVEXITY CONDITIONS AND INTERSECTIONS WITH SMOOTH FUNCTIONS
}

\author{
BY
}

\author{
S. AGRONSKY, A. M. BRUCKNER ${ }^{1}$, M. LACZKOVICH AND D. PREISS
}

\begin{abstract}
A continuous function that agrees with each member of a family $\mathscr{F}$ of smooth functions in a small set must itself possess certain desirable properties. We study situations that arise when $\mathscr{F}$ consists of the family of polynomials of degree at most $n$, as well as certain larger families and when the small sets of agreement are finite. The conclusions of our theorems involve convexity conditions. For example, if a continuous function $f$ agrees with each polynomial of degree at most $n$ in only a finite set, then $f$ is $(n+1)$-convex or $(n+1)$-concave on some interval. We consider also certain variants of this theorem, provide examples to show that certain improvements are not possible and present some applications of our results.
\end{abstract}

1. Introduction. We shall examine the extent to which certain theorems related to monotonicity extend to convexity and, more generally, $n$-convexity (monotonicity itself can be viewed as 1-convexity). By a theorem of E. Čech [2], if a continuous function $f$ defined in an interval takes every value in finitely many points, then $f$ is monotonic in some subinterval. Our main result is the following generalization of Čech's theorem: If the graph of $f$ intersects the graph of every polynomial of degree at most $n$ in finitely many points, then $f$ is $(n+1)$-convex or $(n+1)$-concave in some subinterval (Theorem 13). Čech's result is the case of $n=0$. For the case of $n=1$ we give a separate, elementary proof (Theorem 1 ). In the general theorem, in fact, we prove more: We suppose only that the set $\{x ; f(x)=p(x)\}$ does not have any bilateral point of accumulation for any polynomial $p$ of degree $\leq n$. For $n=0$ this was known. Moreover, if $f$ takes every value on a countable set, then $f$ is monotonic on some subinterval (see [4 or 6]). However, the complete analogue of this theorem does not hold in general. There exists a function $f \in C[a, b]$, the graph of which meets every line in a countable set, but $f$ is convex or concave on no subinterval of $[a, b]$ (Theorem 6).

Čech's theorem can be generalized in another direction: If $f$ takes every value in finitely many points, then every closed set has a portion on which $f$ is monotonic, and $[a, b]$ can be decomposed into countably many subsets on each of which $f$ is

Received by the editors June 19, 1984.

1980 Mathematics Subject Classification. Primary 26A48, 26A51.

Key words and phrases. Monotonicity, $n$-convexity.

${ }^{1}$ The work of this author was supported in part by a grant from the NSF. The work was completed while the third and fourth listed authors were in residence at the University of California at Santa Barbara as participants in a special year in Real Analysis. 
monotonic. (This follows from Čech's results or can easily be proved directly.) We show that, once again, the analogous statement for $n=1$ fails to hold even if the graph of $f$ meets every line in at most five points (Theorem 5). We also examine the cases when the graph of $f$ meets every line in at most three or at most four points, respectively (Theorems 3 and 4).

The positive results will allow us to study the following more general problem. Let $f$ be a continuous function defined on $[a, b]$ and suppose that the graph of $f$ intersects the graph of each member of a given class of smooth functions in a small set. What kind of regularity properties does it impose on the function $f$ ?

We consider the following hierarchy of smooth functions:

$$
P_{0} \subset P_{1} \subset \cdots \subset P_{n} \subset \cdots \subset A \subset C^{\infty} \subset \cdots \subset C^{n} \subset \cdots \subset C^{1}
$$

where $P_{n}$ denotes the class of polynomials of degree at most $n$ (in particular, $P_{0}$ denotes the class of constant functions), and $A, C^{\infty}$ and $C^{n}$ denote the classes of functions which are analytic, infinitely differentiable and $n$ times continuously differentiable on $[a, b]$, respectively.

For example, it follows immediately from Theorem 13 that if $\{x ; f(x)=p(x)\}$ does not have any bilateral point of accumulation for every $p \in P_{n}$, then there is a subinterval on which $f$ is $n-1$ times continuously differentiable (Corollary 14).

Now suppose that $\{x ; f(x)=g(x)\}$ is finite for every $g \in A$. What regularity properties does this condition imply? The problem of the existence of a function $f$ with this property was posed by S. M. Ulam and was solved by Z. Zahorski [10]. Ulam, by mistake, refers to [10] as a proof of the nonexistence of such a function [7, p. 75]. In fact, Zahorski realized that if $f \in C^{\infty}$ and the radius of convergence of the Taylor series of $f$ is zero at every point of $[a, b]$, then $\{x ; f(x)=g(x)\}$ is isolated and, since closed, therefore finite. It should be mentioned that the first example of such a function was constructed by $\mathrm{H}$. Cartan [1, p. 1006]. The problem, whether any function $f$ such that $\{x ; f(x)=g(x)\}$ is finite for each $g \in A$ is infinitely differentiable on a subinterval of $[a, b]$, remains open. We prove, however, that there is no $f \in C[a, b]$ for which $\{x ; f(x)=g(x)\}$ is finite for every $g \in C^{\infty}$ (Corollary 16). (In other words, if $\{x ; f(x)=g(x)\}$ is finite for each $g \in C^{\infty}$, then this imposes the strongest regularity condition, namely the nonexistence of $f$.)

As for countable intersections, we prove that there is no $f \in C[a, b]$ such that $\{x ; f(x)=g(x)\}$ is countable for every $g \in C^{1}$ (Corollary 21). However, the problem of existence of a function $f \in C[a, b]$ such that $\{x ; f(x)=g(x)\}$ is countable for every $g \in C^{2}$ (or $g \in C^{\infty}$ ) remains unsolved.

We first consider, in $\$ 2$, ordinary convexity and intersections with lines. Then in $\$ 3$ we consider higher order convexity and intersections with polynomials and $C^{\infty}$ functions. In $\$ 4$ we deal with analogous problems concerning continuous functions defined on nowhere dense perfect sets.

Finally, in $\$ 5$ we discuss the following problem closely related to our topic. Let $f \in C[a, b]$ be arbitrary. Does there exist a nonempty perfect set on which $f$ is $n$-convex or $n$-concave? 


\section{Ordinary convexity.}

THEOREM 1. Let $f \in C[a, b]$ and suppose that $\{x ; f(x)=g(x)\}$ is finite for every $g \in P_{1}$. Then there is a subinterval of $[a, b]$ on which $f$ is either convex or concave.

LeMma 2. Let $I$ be a subinterval of $[a, b]$ and suppose that $A$ is an everywhere dense subset of $I$ such that for every $x_{0} \in A$ there exists a linear function $l \in P_{1}$ with $l\left(x_{0}\right)=f\left(x_{0}\right)$ and $l(x) \leq f(x)(x \in I)$. Then $f$ is convex on $I$.

Proof. Suppose $f$ is not convex on $I$. Then there are points $x, y, z \in I, x<y<z$, such that $f(y)>u(y)$, where $u$ denotes the linear function satisfying $u(x)=f(x)$ and $u(z)=f(z)$. Since $f$ is continuous, there is a $\delta>0$ such that for every $x_{0} \in(y-\delta, y+\delta)$, if $l$ is a linear function with $l\left(x_{0}\right)=f\left(x_{0}\right)$ then either $l(x)>$ $f(x)$ or $l(z)>f(z)$. Thus $A \cap(y-\delta, y+\delta)=\varnothing$, contradicting our assumption.

Proof of Theorem 1. First we observe that, under the conditions of the theorem, the finite or infinite right-hand side derivative $f_{+}^{\prime}(x)$ exists for every $x \in[a, b)$. In fact, if $\underline{D}_{+} f\left(x_{0}\right)<\bar{D}_{+} f\left(x_{0}\right)$ holds for an $x_{0} \in[a, b]$, then the set

$$
\left\{x \in\left(x_{0}, b\right] ; f(x)=f\left(x_{0}\right)+m\left(x-x_{0}\right)\right\}
$$

is infinite for every $\underline{D}_{+} f\left(x_{0}\right)<m<\bar{D}_{+} f\left(x_{0}\right)$ which is impossible. We put

$$
\begin{aligned}
& B_{+}=\left\{x \in[a, b) ; f_{+}^{\prime}(x)=+\infty\right\}, \\
& B_{-}=\left\{x \in[a, b) ; f_{+}^{\prime}(x)=-\infty\right\} \text { and } \\
& B=B_{+} \cup B_{-} .
\end{aligned}
$$

If $x_{0} \in[a, b) \backslash B$, then we define $g_{x_{0}}(x)=f\left(x_{0}\right)+f_{+}^{\prime}\left(x_{0}\right)\left(x-x_{0}\right)$. Let

$$
\begin{array}{r}
A_{-+}^{n}=\left\{x_{0} \in[a, b) \backslash B ; f(x)<g_{x_{0}}(x)\left(x_{0}-1 / n<x<x_{0}\right)\right. \\
\text { and } \left.f(x)>g_{x_{0}}(x)\left(x_{0}<x<x_{0}+1 / n\right)\right\} ; \\
A_{--}^{n}=\left\{x_{0} \in[a, b) \backslash B ; f(x)<g_{x_{0}}(x)\left(x_{0}-1 / n<x<x_{0}+1 / n, x_{0} \neq x\right)\right\} ; \\
A_{++}^{n}=\left\{x_{0} \in[a, b) \backslash B ; f(x)>g_{x_{0}}(x)\left(x_{0}-1 / n<x<x_{0}+1 / n, x_{0} \neq x\right)\right\} ; \\
A_{+-}^{n}=\left\{x_{0} \in[a, b) \backslash B ; f(x)>g_{x_{0}}(x)\left(x_{0}-1 / n<x<x_{0}\right)\right. \\
\text { and } \left.f(x)<g_{x_{0}}(x)\left(x_{0}<x<x_{0}+1 / n\right)\right\} .
\end{array}
$$

Since $\left\{x ; f(x)=g_{x_{0}}(x)\right\}$ is finite, it follows that

$$
B \cup \bigcup_{n=1}^{\infty}\left(A_{-+}^{n} \cup A_{--}^{n} \cup A_{++}^{n} \cup A_{+-}^{n}\right)=[a, b)
$$

and hence one of these sets is dense in a subinterval. If $A_{++}^{n}$ is dense in a subinterval of length smaller than $1 / n$, then, by Lemma $2, f$ is convex on that subinterval. Similarly, if $A_{--}^{n}$ is dense in an interval, then $f$ is concave on a subinterval.

Hence it is enough to prove that $A_{-+}^{n} \cup B_{-}$and $A_{+-}^{n} \cup B_{+}$are nowhere dense. Suppose that $A_{-+}^{n} \cup B_{-}$is dense in $[\alpha, \beta]$ and that $\beta-\alpha<1 / n$. Subtracting a linear function (which does not change the sets $A_{-+}^{n}$ and $B_{-}$) we can suppose $f(\alpha)=f(\beta)=$ 0 . If $x_{0} \in A_{-+}^{n} \cap(\alpha, \beta)$, then $g_{x_{0}}(\alpha)>0>g_{x_{0}}(\beta)$ and hence $f_{+}^{\prime}\left(x_{0}\right)<0$. The same is 
true if $x_{0} \in B_{-}$and thus $f_{+}^{\prime}(x)<0$ holds on a dense subset of $(\alpha, \beta)$. Since $\{x ; f(x)=g(x)\}$ is finite for every linear $g$, then in particular, $\{x \in[\alpha, \beta] ; f(x)=$ $y\}$ is finite for every $y$. By the theorem of Čech [2], there is an everywhere dense, open set $G \subset(\alpha, \beta)$ such that $f$ is strictly increasing or strictly decreasing on every component of $G$ and, if $G \neq(\alpha, \beta),(\alpha, \beta) \backslash G$ contains an isolated point at which $f$ attains a strict local extremum. In this case, however, $G$ would contain an interval on which $f$ is strictly increasing which is impossible since $f_{+}^{\prime}(x)<0$ holds on a dense set. Thus $G=(\alpha, \beta)$ and hence $f$ is strictly monotonic on $[\alpha, \beta]$, contradicting $f(\alpha)=f(\beta)$. Therefore $A_{-+}^{n} \cup B_{-}$is nowhere dense and the proof of the theorem is complete.

Our next three theorems are concerned with continuous functions, the graphs of which intersect every line in at most $k$ points ( $k$ is finite). Before stating our theorems, we collect the analogous results about continuous functions taking every value in at most $k$ points.

(i) If $\operatorname{card}\{x ; f(x)=y\} \leq 1$ for every $y$, then $f$ is strictly monotonic on $[a, b]$.

(ii) If $\operatorname{card}\{x ; f(x)=y\} \leq 2$ for every $y$, then $[a, b]$ can be decomposed into three subintervals on each of which $f$ is strictly monotonic.

(iii) There is an $f \in C[a, b]$ such that $\operatorname{card}\{x ; f(x)=y\} \leq 3$ for every $y$ and $[a, b]$ cannot be decomposed into countably many subintervals on each of which $f$ is monotonic.

(iv) If $\{x ; f(x)=y\}$ is finite for every $y$, then $[a, b]$ can be decomposed into countably many subsets on each of which $f$ is monotonic.

(i) and (ii) are easily proved. (iv) is a consequence of the fact we mentioned earlier: that, if $\{x ; f(x)=y\}$ is finite for every $y$, then every closed set has a portion on which $f$ is monotonic. As for (iii), consider the following example.

Let $C$ denote the Cantor ternary set in $[0,1]$ and let $\left\{\left(a_{i}, b_{i}\right)\right\}_{i=1}^{\infty}$ be the sequence of intervals contiguous to $C$. If $x \in C$ then we define $f(x)=x$. In $\left[a_{i}, b_{i}\right]$ we define $f$ such that $f\left(a_{i}\right)=a_{i}, f\left(b_{i}\right)=b_{i}, a_{i}<f(x)<b_{i}\left(x \in\left(a_{i}, b_{i}\right)\right)$, and $f$ is strictly increasing on $\left[a_{i}, a_{i}+\frac{1}{3}\left(b_{i}-a_{i}\right)\right]$ and on $\left[b_{i}-\frac{1}{3}\left(b_{i}-a_{i}\right), b_{i}\right]$ and strictly decreasing on $\left[a_{i}+\frac{1}{3}\left(b_{i}-a_{i}\right), b_{i}-\frac{1}{3}\left(b_{i}-a_{i}\right)\right]$. Then $f$ is continuous on $[0,1]$, $\operatorname{card}\{x ; f(x)=y\} \leq 3$ for every $y$ and $(\alpha, \beta) \cap C=\varnothing$ whenever $f$ is monotonic on $(\alpha, \beta)$. This implies that (iii) is true.

These remarks raise many questions about the functions whose graphs meet the graph of every $p \in P_{n}$ in at most $k$ points. We only consider the case of $n=1$. Our first observation is that if the graph of $f \in C[a, b]$ meets every line in at most two points, then $f$ is convex or concave on $[a, b]$.

THEOREM 3. Let $f \in C[a, b]$ and suppose that the graph of $f$ intersects every line in at most three points. Then $[a, b]$ can be decomposed into finitely many subintervals on each of which $f$ is either convex or concave.

Proof. It is enough to show that for every $c \in[a, b]$ there is $\delta>0$ such that $f$ is either convex or concave on each of the intervals $[c-\delta, c] \cap[a, b]$ and $[c, c+\delta] \cap$ $[a, b]$. Let $c \in[a, b)$ be fixed; we prove that $f$ is convex or concave in some right-hand side neighborhood of $c$. Let $K$ denote the convex hull of the graph of $f$ 
over the interval $[c, b]$ and let $\phi(x)=\min \{y ;(x, y) \in K\}$ for $x \in[c, b]$. Then $\phi$ is convex and continuous on $[c, b]$. Let $G$ denote the union of those open subintervals of $(c, b)$ on which $\phi$ is linear. If $G \cap[c, c+\delta]=\varnothing$ for some $0<\delta<b-c$, then $f$ is convex on $[c, c+\delta]$, and thus we can suppose $G \cap[c, c+\delta] \neq \varnothing$ for every $\delta>0$. We prove that there is $z \in(c, b)$ such that $(c, z) \subset G$. Suppose this is not true. Then there is a component $(\alpha, \beta)$ of $G$ for which $c<\alpha<\beta<b$. Let $l$ denote the linear function which is equal to $\phi$ on $(\alpha, \beta)$. Then $y=l(x)$ is a support line of the graph of $f$ with $l(\alpha)=f(\alpha), l(\beta)=f(\beta)$. This easily implies that $f(x)=l(x)+\varepsilon$ has at least four solutions if $\varepsilon>0$ is small enough. This contradicts our assumption. Hence there is $x_{1} \in(c, b]$ such that $\left(c, x_{1}\right)$ is a component of $G$. In particular, $\phi$ is linear on $\left[c, x_{1}\right]$ and, if $l_{1}$ denotes the linear function for which $l_{1}(c)=f(c)$ and $l_{1}\left(x_{1}\right)=f\left(x_{1}\right)$, then $f(x) \geq l_{1}(x)$ for every $x \in\left[c, x_{1}\right]$. Now suppose that $f$ is not concave in any right-hand side neighborhood of $c$. Then, by a similar argument, we can find a point $x_{2} \in\left(c, x_{1}\right]$ such that $f(x) \leq l_{2}(x)$ for every $x \in\left[c, x_{2}\right]$, where $l_{2}$ denotes the linear function satisfying $l_{2}(c)=f(c)$ and $l_{2}\left(x_{2}\right)=f\left(x_{2}\right)$. Since $f$ is not linear on $\left[c, x_{2}\right]$, we have $f\left(x_{2}\right)>l_{1}\left(x_{2}\right)$ and $x_{2}<x_{1}$. Finally, if $f$ is not convex in any right-hand side neighborhood of $c$, then we get a point $x_{3} \in\left(c, x_{2}\right)$ such that $f(x) \geq l_{3}(x)$ for every $x \in\left[c, x_{3}\right]$, where $l_{3}$ is the linear function with $l_{3}(c)=f(c)$ and $l_{3}\left(x_{3}\right)=f\left(x_{3}\right)$. Since $f$ is not linear on $\left[c, x_{3}\right]$, we have $f\left(x_{3}\right)<l_{2}\left(x_{3}\right)$. Now it is easy to check that $f(x)=l_{3}(x)+\varepsilon$ has a solution in each of the intervals $\left(x_{3}, x_{2}\right)$ and $\left(x_{2}, x_{1}\right)$ and has at least two solutions in $\left(c, x_{3}\right)$ if $\varepsilon>0$ is small enough. Thus the graph of $f$ meets the line $y=l_{3}(x)+\varepsilon$ in at least four points which is impossible. This contradiction proves the theorem.

We remark that, by a refinement of the argument above, one can prove that if $\operatorname{card}\{x ; f(x)=g(x)\} \leq 3$ for every $g \in P_{1}$, then $[a, b]$ can be decomposed into five subintervals on each of which $f$ is convex or concave.

THEOREM 4. There exists $g \in C[a, b]$ such that the graph of $g$ meets every line in at most four points and $[a, b]$ cannot be decomposed into countably many subintervals on each of which $g$ is either convex or concave.

Proof. Let $f \in C[a, b]$ be such that $\operatorname{card}\{x ; f(x)=y\} \leq 3$ for every $y$ and $[a, b]$ cannot be decomposed into countably many subintervals on each of which $f$ is monotonic. Then

$$
g(x)=\int_{a}^{x} f(t) d t
$$

obviously satisfies our requirements.

THEOREM 5. Let $C$ denote the Cantor ternary set. There exists a continuous function $f$ defined on $[0,1]$ such that

(i) $f$ is strictly increasing,

(ii) $f$ is Lipschitz,

(iii) the graph of $f$ meets every line in at most 5 points,

(iv) $f$ is neither convex nor concave on any portion of $C$,

(v) $[0,1]$ cannot be decomposed into countably many subsets on each of which $f$ is either convex or concave. 
Proof. Let $\phi$ be a function defined on $[0,1]$ and having the following property: Whenever $J$ is a bounded interval contiguous to $C$, then $\phi$ is strictly increasing on $J$ and $|J|^{4}<\phi(x)<2|J|^{4}$ for every $x \in J$. Let $f(x)=\int_{0}^{x} \phi(t) d t(x \in[0,1])$. Then properties (i) and (ii) are obvious. Since $f^{\prime}(x)=0$ at every bilateral point of accumulation of $C$, (i) implies (iv). In order to prove (v), suppose that $[0,1]=\bigcup_{k=1}^{\infty} A_{k}$ and that $f$ is either convex or concave on each $A_{k}$. Now $C \subset \bigcup_{k=1}^{\infty} A_{k}$ so, by the Baire category theorem, there is a $k$ such that $A_{k}$ is dense in a portion of $C$. By the continuity of $f, f$ is convex or concave on this portion which contradicts (iv).

Now we prove (iii). Suppose that (iii) is not true. Then there is a set $A=\left\{p_{1}<p_{2}\right.$ $\left.<\cdots<p_{6}\right\} \subset[0,1]$ and $\alpha \in \mathbf{R}$ such that $(u-v)^{-1} \int_{v}{ }^{u} \phi(t) d t=\alpha$ for all $u, v \in A$, $u \neq v$. Let $J_{0}=(a, b)$ be the largest interval contiguous to $C$ which is contained in $\left(p_{1}, p_{6}\right)$. Let $J_{3}=(2 a-b, a)$ and $J_{4}=(b, 2 b-a)$, and let $J_{1}$ and $J_{2}$ be intervals contiguous to $C$ such that $\{2 a-b, 2 b-a\} \subset \bar{J}_{1} \cup \bar{J}_{2}$ and $\left|J_{2}\right| \geq\left|J_{1}\right| \geq\left|J_{0}\right|$. Then $\left|J_{0}\right|=\left|J_{3}\right|=\left|J_{4}\right|$; either $\left|J_{1}\right|=3\left|J_{0}\right|$ or $J_{1}$ is unbounded and $\left|J_{2}\right| \geq 9\left|J_{0}\right|$.

To arrive at a contradiction, we first use the monotonicity of $\phi$ on intervals contiguous to $C$ to infer that $\operatorname{card}\left(J_{i} \cap A\right) \leq 2$ for $i=0,1,2$. As a consequence we get two points $u, v \in A, u<v$, such that $[u, v] \cap\left(J_{1} \cup J_{2}\right)=\varnothing$. Since $\phi(x) \leq 2\left|J_{0}\right|^{4}$ for a.e. $x \in(u, v)$ and $\phi(y) \geq\left(3\left|J_{0}\right|\right)^{4}>2\left|J_{0}\right|^{4}$ for every $y \in J_{1} \cup J_{2}$, we have $\operatorname{card}\left(\bar{J}_{1} \cap A\right) \leq 1$ and $\operatorname{card}\left(\bar{J}_{2} \cap A\right) \leq 1$.

Next we prove

$$
\frac{1}{p_{6}-p_{1}} \int_{p_{1}}^{p_{6}} \phi(x) d x \geq \frac{1}{12}\left|J_{0}\right|^{4} .
$$

Indeed, if $p_{6}-p_{1} \leq 12\left|J_{0}\right|$, then

$$
\frac{1}{p_{6}-p_{1}} \int_{p_{1}}^{p_{6}} \phi(x) d x \geq \frac{1}{p_{6}-p_{1}} \int_{J_{0}} \phi(x) d x \geq \frac{1}{12\left|J_{0}\right|} \cdot\left|J_{0}\right|^{5}=\frac{1}{12}\left|J_{0}\right|^{4} .
$$

If $p_{6}-p_{1}>12\left|J_{0}\right|$ and $\left(p_{1}, p_{6}\right) \cap J_{0}=I$, then $|I| \geq 6\left|J_{0}\right|$ and $p_{6}-p_{1} \leq 2|I|$. Thus

$$
\begin{aligned}
\frac{1}{p_{6}-p_{1}} \int_{p_{1}}^{p_{6}} \phi(x) d x & \geq \frac{1}{p_{6}-p_{1}} \int_{I} \phi(x) d x \geq \frac{1}{2|I|} \int_{I} \phi(x) d x \\
& \geq \frac{1}{2}\left(9\left|J_{0}\right|\right)^{4}>\frac{1}{12}\left|J_{0}\right|^{4} .
\end{aligned}
$$

On the other hand, for $y \in J_{3} \cup J_{4}$ we have

$$
\phi(y) \leq 2\left(\frac{1}{3}\left|J_{0}\right|\right)^{4}=\frac{2}{81}\left|J_{0}\right|^{4}<\frac{1}{12}\left|J_{0}\right|^{4}
$$

and hence $\operatorname{card}\left(\bar{J}_{3} \cap A\right) \leq 1$ and $\operatorname{card}\left(\bar{J}_{4} \cap A\right) \leq 1$.

From these results it follows that the only possibility is $\operatorname{card}\left(J_{i} \cap A\right)=1$ for $i=1,2,3,4$ and card $\left(J_{0} \cap A\right)=2$. In particular, $p_{2} \in \bar{J}_{3}$ and $p_{3}, p_{4} \in J_{0}$. But this is impossible, since then

$$
\frac{1}{p_{3}-p_{2}} \int_{p_{2}}^{p_{3}} \phi(t) d t \leq \phi\left(p_{3}\right)<\frac{1}{p_{4}-p_{3}} \int_{p_{3}}^{p_{4}} \phi(t) d t
$$

This contradiction completes the proof of (iii). 
Before we state our theorem we need some definitions. A function $f:[a, b] \rightarrow \mathbf{R}$ is said to be regulated if the finite limits $f(x+)=\lim _{y \rightarrow x+} f(y)$ and $f(z-)=$ $\lim _{n \rightarrow z-} f(w)$ exist for every $x \in[a, b)$ and $z \in(a, b]$, respectively. Every regulated function is bounded and the class of regulated functions on $[a, b]$, endowed with the sup norm, constitutes a Banach space $\mathscr{R}$. We say that a property $P$ is typical in $\mathscr{R}$ if the set of functions $f \in \mathscr{R}$ not having property $P$ is of first category.

THEOREM 6. A typical $f \in \mathscr{R}$ has the following properties.

(i) If $x, y \in[a, b], x \neq y$, then $f(x+) \neq f(y+), f(x+) \neq f(y-), f(x-) \neq$ $f(y+), f(x-) \neq f(y-)$;

(ii) $f(x+)$ is not monotonic on any subinterval of $[a, b]$.

If $f \in \mathscr{R}$ has properties (i) and (ii) and

$$
g(x)=\int_{a}^{x} f(t) d t \quad(x \in[a, b])
$$

then

(a) $g$ is Lipschitz;

(b) $g$ is neither convex nor concave in any subinterval of $[a, b]$;

(c) the graph of $g$ intersects every line in a countable set.

Proof. We put

$$
\begin{aligned}
E_{n}=\{f \in \mathscr{R} ; \text { there are } x, y \in[a, b],|x-y| \geq 1 / n \\
\quad \text { such that }\{f(x-), f(x+)\} \cap\{f(y-), f(y+)\} \neq \varnothing\} \quad(n=1,2, \ldots) .
\end{aligned}
$$

Then $E_{n}$ is closed for every $n$. In fact, let $f_{k} \in E_{n}(k=1,2, \ldots), f_{k} \rightarrow f$ uniformly, and let $x_{k}, y_{k} \in[a, b]$ such that $\left|x_{k}-y_{k}\right| \geq 1 / n$ and $\left\{f_{k}\left(x_{k}-\right), f_{k}\left(x_{k}+\right)\right\} \cap$ $\left\{f_{k}\left(y_{k}-\right), f_{k}\left(y_{k}+\right)\right\} \neq \varnothing(k=1,2, \ldots)$. Selecting a subsequence, if necessary, we can assume $x_{k} \rightarrow x, y_{k} \rightarrow y$. Then $|x-y| \geq 1 / n$ and if, say, $x_{k}>x$ and $y_{k}>y$ for infinitely many $k$, then $f(x+)=f(y+)$. Hence $f \in E_{n}$ and thus $E_{n}$ is closed.

Now we show that $E_{n}$ is nowhere dense for every $n$; this will prove that a typical $f \in \mathscr{R}$ has property (i). Let $g \in \mathscr{R}$ and $\varepsilon>0$ be arbitrary. It is easy to see that there is a partition $a=x_{0}<x_{1}<\cdots<x_{k}=b$ such that $x_{i}-x_{i-1}<1 / n$ and the oscillation of $g$ on $\left(x_{i-1}, x_{i}\right)$ is smaller than $\varepsilon$ for every $i=1, \ldots, k$. Now let $c_{1}, \ldots, c_{k}$ be distinct real numbers such that $\left|g(x)-c_{i}\right|<\varepsilon$ for every $x \in\left(x_{i-1}, x_{i}\right)$, $i=1, \ldots, k$. Then the function $h$ defined by $h(x)=c_{i}\left(x \in\left(x_{i-1}, x_{i}\right), i=1, \ldots, k\right)$ and $h\left(x_{i}\right)=g\left(x_{i}\right)(i=0,1, \ldots, k)$ is regulated, $|h-g|<\varepsilon$ and $h \notin E_{n}$. This proves that $E_{n}$ does not contain any ball and hence, being closed, $E_{n}$ is nowhere dense.

Let $\left\{I_{n}\right\}$ denote the sequence of open subintervals of $[a, b]$ with rational endpoints. Let $F_{n}=\left\{f \in \mathscr{R} ; f(x+)\right.$ is monotonic in $\left.I_{n}\right\}$. It is easy to check that $F_{n}$ is closed and nowhere dense in $\mathscr{R}$ for every $n$ and this proves the first half of the theorem.

Now let $g(x)=-\int_{a}^{x} f(t) d t$, where $f \in \mathscr{R}$ satisfies (i) and (ii). Then (a) is obvious and (b) follows from (ii). Let $c, d$ be given real numbers and let $L=\{x \in$ $[a, b] ; g(x)=c x+d\}$. Since $f$ is regulated, we have $g^{\prime}(x+)=f(x+)$ and $g^{\prime}(x-)=f(x-)$ for every $x$. Thus, if $x$ is a right- (left-)hand side point of 
accumulation of $L$, then $g^{\prime}(x+)=f(x+)=c\left(g^{\prime}(x-)=f(x-)=c\right)$. (i) implies that $L$ can have at most one point of accumulation and thus $L$ is countable. This completes the proof.

3. $n$-convex functions. This section is devoted to Theorems 13 and 15 . We begin with some definitions and notation.

Let $f$ be defined on a set $E \subset \mathbf{R}$. For every $n \geq 0$, the $n$th divided difference of $f$ at the distinct points $x_{0}, \ldots, x_{n} \in E$ is defined by

$$
V\left(f ; x_{0}, \ldots, x_{n}\right)=\sum_{i=0}^{n} \frac{f\left(x_{i}\right)}{w^{\prime}\left(x_{i}\right)},
$$

where $w(x)=\prod_{j=0}^{n}\left(x-x_{j}\right)$. The function $f$ is said to be $n$-convex on $E$ if $V\left(f ; x_{0}, \ldots, x_{n}\right) \geq 0$ whenever $x_{0}, \ldots, x_{n}$ are distinct elements of $E$. We say that $f$ is $n$-concave if $-f$ is $n$-convex. Thus $f$ is 0 -convex if $f \geq 0$ on $E, f$ is 1 -convex if $f$ is increasing on $E$ and $f$ is 2-convex if $f$ is convex on $E$. If $E$ is an open interval and $n \geq 2$, then $f$ is $n$-convex on $E$ if and only if $f$ is $n-2$ times continuously differentiable and $f^{(n-2)}$ is convex on $E$.

Let $\omega$ be a nonnegative finite Borel measure on $[0,1]$ such that $\omega(\{0\})=0$ and $\int_{0}^{1} t d \omega(t)=1$.

If $f$ is bounded and Borel measurable in a neighborhood of $x$, then we put $\bar{D}_{+}^{\omega} f(x)=\inf \{r \in \mathbf{R}$; there is a $\delta>0$ such that the set

$$
\left.\left\{h \in(0, \delta) ; \frac{1}{h} \int_{0}^{1}(f(x+t h)-f(x)) d \omega(t)>r\right\} \text { is countable }\right\} .
$$

The definitions of $\bar{D}_{-}^{\omega} f(x), \underline{D}_{+}^{\omega} f(x)$ and $\underline{D}_{-}^{\omega} f(x)$ are analogous. We define

$$
\bar{D}^{\omega} f(x)=\max \left(\bar{D}_{-}^{\omega} f(x), \bar{D}_{+}^{\omega} f(x)\right) \quad \text { and } \quad \underline{D}^{\omega} f(x)=\min \left(\underline{D}_{-}^{\omega} f(x), \underline{D}_{+}^{\omega} f(x)\right) \text {. }
$$

It is easy to check that

$$
\underline{D}_{+} f(x) \leq \underline{D}_{+}^{\omega} f(x) \leq \bar{D}_{+}^{\omega} f(x) \leq \bar{D}_{+} f(x)
$$

and

$$
\underline{D}_{-} f(x) \leq \underline{D}_{-}^{\omega} f(x) \leq \bar{D}_{-}^{\omega} f(x) \leq \bar{D}_{-} f(x) .
$$

We shall say that $D^{\omega} f$ does not change sign at $x$ if there is $\delta>0$ such that at least one of the following statements holds.

(i) $\int_{0}^{1}(f(x+t h)-f(x)) d \omega(t) \leq 0$ for all but countably many $h \in(0, \delta)$.

(ii) $\int_{0}^{1}(f(x+t h)-f(x)) d \omega(t) \geq 0$ for all but countably many $h \in(0, \delta)$.

(iii) $\int_{0}^{1}(f(x+t h)-f(x)) d \omega(t) \leq 0$ for all but countably many $h \in(-\delta, 0)$.

(iv) $\int_{0}^{1}(f(x+t h)-f(x)) d \omega(t) \geq 0$ for all but countably many $h \in(-\delta, 0)$.

In the following Lemmas 7-11 we suppose that $f$ is a regulated function defined on an interval $[a, b]$ and that $f(x)$ lies between $f(x-)$ and $f(x+)$ for every $x \in(a, b)$.

Denote $C_{+}=\{x \in(a, b) ; f(x)=f(x+)\}, C_{-}=\{x \in(a, b) ; f(x)=f(x-)\}$ and $C=C_{-} \cap C_{+}$. Since $f$ is regulated, $(a, b) \backslash C$ is countable. We define for every $x \in(a, b)$ and $\delta>0$

$$
H_{x}^{\delta}=\{h \in(0, \delta) ; x+t h \in C \text { for } \omega \text {-a.e. } t \in(0,1]\} .
$$


LEMMA 7. $(0, \delta) \backslash H_{x}^{\delta}$ is countable for every $x \in(a, b)$ and $\delta>0$.

Proof. The sets $(a, b) \backslash C$ and $T=\{t \in(0,1] ; \omega(\{t\})>0\}$ are countable and hence so is $(0, \delta) \backslash H_{x}^{\delta} \subset\{(y-x) / t ; y \in(a, b) \backslash C, t \in T\}$.

LEMMA 8. Let $r \in \mathbf{R}, \delta>0$ be given and let $A=\{x \in(a, b)$; there are uncountably many $h \in(0, \delta)$ such that $\left.\int_{0}^{1} f(x+t h) d \omega(t)>r\right\}$, and $B=\{x \in(a, b)$; there are uncountably many $h \in(0, \delta)$ such that $\left.h^{-1} \int_{0}^{1}[f(x+t h)-f(x)] d \omega(t)>r\right\}$. Then

(i) $A$ is open;

(ii) for every $x \in B$ there is $\eta>0$ with $\{y \in(x-\eta, x+\eta)$; $f(y)<f(x)+\eta\} \subset$ B.

Proof. We only prove (i) since the proof of (ii) is similar. Suppose that $A$ is not open. Then there is $x \in A$ and a sequence $x_{n} \rightarrow x, x_{n} \notin A$. Let $K_{n}=\{h \in(0, \delta)$; $\left.\int_{0}^{1} f\left(x_{n}+t h\right) d \omega(t)>r\right\}$ and $K=\bigcup_{n=1}^{\infty} K_{n}$. If $h \in(0, \delta) \cap\left(H_{x}^{\delta} \backslash K\right)$, then

$$
\int_{0}^{1} f(x+t h) d \omega(t)=\lim _{n \rightarrow \infty} \int_{0}^{1} f\left(x_{n}+t h\right) d \omega(t) \leq r .
$$

Since $K$ is countable by the choice of $x_{n}$ and $(0, \delta) \backslash H_{x}^{\delta}$ is countable by Lemma 7 , this contradicts $x \in A$.

LEMMA 9. Let I be a subinterval of $(a, b)$ and suppose that the set $\left\{x \in I ; D^{\omega} f\right.$ does not change sign at $x\}$ is of second category. Then $f$ is monotone on some subinterval of I.

Proof. There is a $\delta>0$ such that one of the alternatives (i)-(iv) holds on a set dense in an open subinterval $J \subset I$. Suppose for example that the set

$E=\left\{x ; \int_{0}^{1}[f(x+t h)-f(x)] d \omega(t) \leq 0\right.$ for all but countably many $\left.h \in(0, \delta)\right\}$

is dense in $J$; we may also assume $|J|<\delta$. Then $\left(C_{-} \cup C_{+}\right) \cap J \subset E$. Indeed, if $x \in C_{+} \cap J$ and $x_{n} \in E, x_{n} \downarrow x$, then we have

$$
\int_{0}^{1}[f(x+t h)-f(x)] d \omega(t)=\lim _{n \rightarrow \infty} \int_{0}^{1}\left[f\left(x_{n}+t h\right)-f\left(x_{n}\right)\right] d \omega(t)
$$

for every $h \in H_{x}^{\delta}$ from which $x \in E$ easily follows.

We prove that for every $x, y \in C \cap J, x<y, f(x) \geq f(y)$ holds. Since $f$ is regulated and $f(z)$ lies inbetween $f(z-)$ and $f(z+)$, this implies that $f$ is decreasing on $J$.

Suppose $x, y \in C \cap J, x<y$, and $f(x)<f(y)$. Let $c=\sup \{u \in[x, y] ; f(u) \leq$ $f(x)\}$. Then $c \in[x, y)$ and

$$
\int_{0}^{1}[f(c+t h)-f(x)] d \omega(t)>0
$$

for every $h \in(0, y-c)$. Since $x \in E$, this proves $c>x$. By Lemma 8(i) there is $0<\eta<c-x$ such that for every $z \in(c-\eta, c+\eta)$

$$
\int_{0}^{1}[f(z+t h)-f(x)] d \omega(t)>0
$$


holds for uncountably many $h \in(0, y-c)$. If there is a $z \in(c-\eta, c)$ with $f(z)<$ $f(x)$, then we have either $f(z-)<f(x)$ or $f(z+)<f(x)$ and hence we can choose a point $u \in(c-\eta, c) \cap C$ with $f(u)<f(x)$. Then

$$
\int_{0}^{1}[f(u+t h)-f(u)] d \omega(t)>\int_{0}^{1}[f(u+t h)-f(x)] d \omega(t)>0
$$

for uncountably many $h \in(0, y-c)$ which contradicts $u \in E$. Hence $f(z) \geq f(x)$ holds for every $z \in(c-\eta, c)$. By the definition of $c$ we can choose a point $v \in(c-\eta, c]$ with $f(v)=f(x)$. Then $f(v-)=f(x)$ or $f(v+)=f(x)$ holds and hence $v \in\left(C_{-} \cup C_{+}\right) \cap J \subset E$. On the other hand,

$$
\int_{0}^{1}[f(v+t h)-f(v)] d \omega(t)=\int_{0}^{1}[f(v+t h)-f(x)] d \omega(t)>0
$$

for uncountably many $h \in(0, y-c)$, which is a contradiction again.

LEMMA 10. The set $\left\{x \in(a, b) ; \bar{D}_{+}^{\omega} f(x) \neq \bar{D}_{-}^{\omega} f(x)\right\}$ is of first category.

Proof. It is enough to prove that the set $F_{r}=\left\{x \in(a, b) ; \bar{D}_{+}^{\omega} f(x)>r>\right.$ $\left.\bar{D}_{-}^{\omega} f(x)\right\}$ is of first category for every rational $r$. Assuming that this is not the case, we find a rational $r$ and an interval $I \subset(a, b)$ such that $F_{r}$ is of second category on each subinterval of $I$. Then Lemma 9 implies that $f-r x$ is decreasing on some open subinterval $J \subset I$. Hence for every $x \in J$ we have

$$
\bar{D}_{+}^{\omega} f(x) \leq \bar{D}_{+} f(x) \leq r,
$$

which is impossible.

LEMMA 11. If $I \subset(a, b)$ is an open interval, $f(x-) \geq f(x) \geq f(x+)$ for every $x \in I$, Int $\left\{x \in I ; \bar{D}^{\omega} f(x)<0\right\}$ is everywhere dense in $I$ and $\bar{D}^{\omega} f(y)>0$ for some $y \in I$, then the set

$$
P=\overline{\left\{x \in I ; \bar{D}^{\omega} f(x)>0\right\}}
$$

is nonempty and perfect and $D^{\omega} f$ changes sign at every point of some residual subset of $P$.

Proof. The set $P$ is obviously nonempty, closed and nowhere dense. We show that if $\bar{D}_{+}^{\omega} f(x)>0$, then $x$ is a right accumulation point of $P$. In fact, if $(x, x+\delta) \cap P$ $=\varnothing$, then $\underline{D}_{+} f(y) \leq \bar{D}^{\omega} f(y) \leq 0$ for every $y \in(x, x+\delta)$. This, together with the condition $f(y+) \geq f(y) \geq f(y-)$, implies that $f$ is decreasing on $[x, x+\delta)$ which is impossible by $\bar{D}_{+} f(x) \geq \bar{D}_{+}^{\omega} f(x)>0$. Similarly, if $\bar{D}_{-}^{\omega} f(x)>0$, then $x$ is a left accumulation point of $P$ and hence $P$ is perfect.

Let

$$
\begin{aligned}
B_{n}^{+}=\{x \in I ; & \text { there are uncountably many } h \in(0,1 / n) \\
& \text { such that } \left.h^{-1} \int_{0}^{1}[f(x+t h)-f(x)] d \omega(t)>0\right\} .
\end{aligned}
$$

Then $B_{n}^{+}$contains a subset of $P$ which is dense and open relative to $P$. Indeed, let $J$ be an open interval with $J \cap P \neq \varnothing$. Since there is a point $x \in J \cap P$ with $\bar{D}^{\omega} f(x)>0, f$ is not decreasing on $J$, and hence there is a $y \in J$ with $\underline{D}_{+} f(y)>0$. 
Then $y$ is a right-hand side accumulation point of $P$. By Lemma 8(ii) and since $f(y) \geq f(y+)$, it follows that $B_{n}^{+}$contains a right-hand side neighborhood of $y$, and hence a portion of $P$. This implies that $B_{n}^{+}$and also $B^{+}=\bigcap_{n=1}^{\infty} B_{n}^{+}$is residual in $P$.

Now let

$$
\begin{aligned}
& E_{n}^{+}=\{x \in I ; \text { there are uncountably many } h \in(0,1 / n) \\
& \text { such that } \left.h^{-1} \int_{0}^{1}[f(x+t h)-f(x)] d \omega(t)<0\right\} .
\end{aligned}
$$

Since $f$ is strictly decreasing in every interval contiguous to $P, E_{n}^{+}$contains every right isolated point of $P$. Applying Lemma 8(ii) for the function $-f$, it follows that $E_{n}^{+}$contains a left-hand side neighborhood of every right isolated point of $P$. Therefore $E^{+}=\bigcap_{n=1}^{\infty} E_{n}^{+}$is residual in $P$. It can be shown similarly that the analogously defined sets $B^{-}, E^{-}$are residual in $P$ as well. Obviously, $D^{\omega} f$ changes sign at each point of $B^{+} \cap E^{+} \cap B^{-} \cap E^{-}$.

THEOREM 12. Suppose that $f$ is regulated in $[a, b], f(x)$ lies between $f(x-)$ and $f(x+)$ for each $x \in(a, b)$ and the set of those $x$ for which there is a linear function $g$ such that $D^{\omega}(f-g)$ changes sign at $x$ is countable. Then there is a subinterval of $(a, b)$ on which $f$ is convex or concave.

Proof. First we show that there is a residual subset $G \subset(a, b)$ such that $\bar{D}^{\omega} f(x)=\underline{D}^{\omega} f(x)$ for each $x \in G$ and the functions $\bar{D}^{\omega} f$ and $\underline{D}^{\omega} f$ are continuous at each point of $G$. Assuming that this is not the case, we find $r, s, \in \mathbf{R}, r<s$, and an interval $I$ such that the sets $\left\{x \in I ; \bar{D}^{\omega} f(x)>s\right\}$ and $\left\{x \in I ; \underline{D}^{\omega} f(x)<r\right\}$ are both dense in $I$. From Lemma 8(ii) we deduce that the sets $\left\{x \in I ; \bar{D}^{\omega} f(x) \geq s\right\}$ and $\left\{x \in I ; \underline{D}^{\omega} f(x) \leq r\right\}$ are both residual in $I$. Using Lemma 10 we find that there are uncountably many $x \in I$ such that $\bar{D}_{+}^{\omega} f(x)=\bar{D}_{-}^{\omega} f(x) \geq s$ and $\underline{D}_{+}^{\omega} f(x)=$ $\underline{D}_{-}^{\omega} f(x) \leq r$. But $D^{\omega}\left(f(t)-\frac{1}{2}(r+s) t\right)$ changes sign at each such $x$, which is a contradiction.

Next we show that there is $x_{0} \in G$ such that $-\infty<\underline{D}^{\omega} f\left(x_{0}\right)=\bar{D}^{\omega} f\left(x_{0}\right)<\infty$. In fact, we prove that the sets $U=\left\{x \in G ; \bar{D}^{\omega} f(x)=-\infty\right\}$ and $V=\left\{x \in G ; \bar{D}^{\omega} f(x)\right.$ $=\infty\}=\left\{x \in G ; \underline{D}_{\omega} f(x)=\infty\right\}$ are nowhere dense in $(a, b)$. Suppose this is not true and let $U$ be dense in a subinterval $J$. By Lemma $9, f$ is monotone in some subinterval $J_{1} \subset J$ and since $U$ is dense in $J_{1}, f$ is decreasing in $J_{1}$. Let $z \in J_{1}$ be a point at which $f^{\prime}(z)$ is finite. Since $\operatorname{Int}\left\{x \in J_{1} ; \bar{D}^{\omega} f(x)<f^{\prime}(z)-1\right\}$ is dense in $J_{1}$, we may apply Lemma 11 to the function $h(t)=f(t)-\left(f^{\prime}(z)-1\right) t$. (Since $f$ is decreasing on $J_{1}, h(t-) \geq h(t) \geq h(t+)$ holds in $J_{1}$ and the lemma is applicable.) Thus we get uncountably many points at which $D^{\omega} h$ changes sign, contradicting our assumption on $f$. Hence $U$ and $V$ are nowhere dense and we can choose a point $x_{0} \in G \backslash(U \cup V)$.

Let $I_{0}$ be an open interval containing $x_{0}$ on which $\bar{D}^{\omega} f$ and $\underline{D}^{\omega} f$ are bounded. From Lemma 9 we easily deduce that $f$ is Lipschitz on some subinterval $I_{1} \subset I_{0}$. 
Now we prove that for every open interval $J \subset I_{\mathrm{r}}$, we have

$$
\begin{aligned}
& \sup \left\{\bar{D}^{\omega} f(x) ; x \in J \cap G\right\}=\sup \left\{\bar{D}^{\omega} f(x) ; x \in J\right\} \quad \text { and } \\
& \inf \left\{\bar{D}^{\omega} f(x) ; x \in J \cap G\right\}=\inf \left\{\bar{D}^{\omega} f(x) ; x \in J\right\} .
\end{aligned}
$$

Suppose this is not true and let

$$
\sup \left\{\bar{D}^{\omega} f(x) ; x \in J \cap G\right\}<r<\sup \left\{\bar{D}^{\omega} f(x) ; x \in J\right\} .
$$

Since $f$ is continuous on $J$, we can apply Lemma 11 to the function $f(t)-r t$ on $J$. Thus we get uncountably many points at which $D^{\omega}(f(t)-r t)$ changes sign, which is impossible. A similar argument shows

$$
\inf \left\{\underline{D}^{\omega} f(x) ; x \in J \cap G\right\}=\inf \left\{\underline{D}^{\omega} f(x) ; x \in J\right\}
$$

from which we get

$$
\begin{aligned}
\inf \left\{\bar{D}^{\omega} f(x) ; x \in J\right\} & \geq \inf \left\{\underline{D}^{\omega} f(x) ; x \in J\right\}=\inf \left\{\underline{D}^{\omega} f(x) ; x \in J \cap G\right\} \\
& =\inf \left\{\bar{D}^{\omega} f(x) ; x \in J \cap G\right\} \geq \inf \left\{\bar{D}^{\omega} f(x) ; x \in J\right\},
\end{aligned}
$$

proving the second equality in $(*)$.

For each $x \in G \cap I_{1}$, consider the function $h_{x}(u)=f(u)-\left(\bar{D}^{\omega} f(x)\right) u$. Except for countably many $x \in G \cap I_{1}, h_{x}$ fulfills one of the alternatives (i)-(iv) and hence one of these holds on a second category subset of $G$. Without loss of generality we may assume that it is (ii) and hence that there is an open subinterval $I_{2} \subset I_{1}$ and $\delta>0$ such that $\left|I_{2}\right|<\delta$ and

$$
\int_{0}^{1}\left[f(x+t h)-f(x)-\left(\bar{D}^{\omega} f(x)\right) t h\right] d \omega(t) \geq 0
$$

for every $h \in(0, \delta)$ and $x \in G \cap I_{2}$. (Here we also used the continuity of $f$ on $I_{1}$ and the continuity of $\bar{D}^{\omega}$ on $I_{1} \cap G$.)

We prove that $f$ is convex on $I_{2}$. Since $f$ is Lipschitz, $f$ is the integral of $f^{\prime}$ and $f^{\prime}(x)=\bar{D}^{\omega} f(x)$ at a.e. $x \in I_{2}$. Hence it is enough to show that $\bar{D}^{\omega} f$ is increasing on $I_{2}$. By (*), $\bar{D}^{\omega} f$ is increasing on $I_{2}$ if and only if it is increasing on $I_{2} \cap G$. Therefore if we show that $\bar{D}^{\omega} f$ is increasing on $I_{2} \cap G$, the proof will be finished. Assume, on the contrary, that there are $x, y \in I_{2} \cap G, x<y$, with $\bar{D}^{\omega} f(x)>\bar{D}^{\omega} f(y)$. Let

$$
u=\sup \left\{z \in[x, y] ; \bar{D}^{\omega} f(z) \geq \bar{D}^{\omega} f(x)\right\} .
$$

Then $u \in[x, y)$ since $\bar{D}^{\omega} f$ is continuous at $y$. For every $h \in(0, y-u)$ we have

$$
f(u+h)-f(u)=\int_{u}^{u+h} f^{\prime}(t) d t=\int_{u}^{u+h} \bar{D}^{\omega} f(t) d t<h \cdot \bar{D}^{\omega} f(x)
$$

and hence

$$
\frac{1}{h} \int_{0}^{1}[f(u+t h)-f(u)] d \omega(t)<\bar{D}^{\omega} f(x) .
$$

This implies that $u>x$. Indeed, since $x \in G \cap I_{2}$, by the choice of $I_{2}$ we have

$$
\frac{1}{h} \int_{0}^{1}[f(x+t h)-f(x)] d \omega(t) \geq \bar{D}^{\omega} f(x)
$$


for every $h \in(0, \delta)$. Let $h \in(0, y-u)$ be fixed. Since $f$ is continuous, $(* *)$ implies that there is $\eta>0$ such that

$$
\frac{1}{h} \int_{0}^{1}[f(z+t h)-f(z)] d \omega(t)<\bar{D}^{\omega} f(x)-\eta
$$

for every $z \in(u-\eta, u+\eta)$. By the definition of $u, \sup \left\{\bar{D}^{\omega} f(z) ; z \in(u-\eta, u+\right.$ $\eta)\} \geq \bar{D}^{\omega} f(x)$ and hence, by (*), $\sup \left\{\bar{D}^{\omega} f(z) ; z \in(u-\eta, u+\eta) \cap G\right\} \geq \bar{D}^{\omega} f(x)$. Let $z \in(u-\eta, u+\eta) \cap G$ be such that $\bar{D}^{\omega} f(z)>\bar{D}^{\omega} f(x)-\eta$. Then we have

$$
\frac{1}{h} \int_{0}^{1}[f(z+t h)-f(z)] d \omega(t)<\bar{D}^{\omega} f(x)-\eta<\bar{D}^{\omega} f(z) .
$$

However, $z \in I_{2} \cap G$ and hence

$$
\frac{1}{h} \int_{0}^{1}[f(z+t h)-f(z)] d \omega(t) \geq \bar{D}^{\omega} f(z)
$$

for every $h \in(0, \delta)$, which is a contradiction. Thus the proof of the theorem is complete.

THEOREM 13. Let $f$ be continuous on $[a, b]$ and suppose that for every polynomial $p \in P_{n}$ the set $\{x ; f(x)=p(x)\}$ does not have a bilateral point of accumulation. Then $f$ is $(n+1)$-convex or $(n+1)$-concave in a subinterval of $(a, b)$.

Proof. We prove by induction on $n$. If $n=0$ then the assertion is well known (see [6]). Or, we may apply Lemma 9 with $\omega$ being the Dirac measure concentrated at $t=1$.

If $n=1$, then let $\omega$ denote the Dirac measure again. Let $g(u)=c u+d$ be a linear function and let $x \in(a, b)$ be fixed. Then

$$
\int_{0}^{1}[f(x+t h)-g(x+t h)-f(x)+g(x)] d \omega(t)=f(x+h)-f(x)-c h
$$

for every $h$ with $x+h \in(a, b)$. Since $x$ is not a bilateral point of accumulation of the set $\{u ; f(u)=f(x)+c(u-x)\}$ and $f$ is continuous, $f(x+h)-f(x)-c h$ does not change sign in a one-sided neighborhood of the origin. Therefore $D^{\omega}(f-g)$ does not change sign at any point of $(a, b)$ and hence, by Theorem $12, f$ is 2 -convex or 2-concave in a subinterval of $(a, b)$.

Now suppose that $n \geq 2$ and that the assertion has been proved for $n-1$. This implies that $f$ is $n$-convex or $n$-concave on an open subinterval $I \subset(a, b)$. Therefore $f^{(n-2)}$ is convex or concave and $f_{+}^{(n-1)}$ is finite, regulated and continuous from the right in $I$.

We prove that $f_{+}^{(n-1)}$ is convex or concave in a subinterval $J \subset I$; this will imply that $f$ is $(n+1)$-convex or $(n+1)$-concave in $J$.

By Theorem 12, in order to prove this, it is enough to find a Borel measure $\omega$ with $\omega(\{0\})=0$ and $\int_{0}^{1} t d \omega(t)=1$ and such that for every linear $g, D^{\omega}\left(f_{+}^{(n-1)}-g\right)$ does not change sign at any point of $I$. We show that the measure $\omega$ defined by

$$
\omega(E)=(n-1) n \int_{E}(1-t)^{n-2} d t \quad(E \subset[0,1] \text { Borel })
$$

has this property. 
It is well known (and easy to prove by induction on $k$ ) that if $f^{(k-1)}$ is absolutely continuous on $[x, x+h]$, then

$$
f(x+h)-\sum_{i=0}^{k-1} \frac{f^{(i)}(x)}{i !} h^{i}=\frac{h^{k}}{(k-1) !} \int_{0}^{1} f^{(k)}(x+t h)(1-t)^{k-1} d t .
$$

Let $x \in I$ be fixed and let $x+h \in I$. Since every convex or concave function is absolutely continuous, we have

$$
f(x+h)-p_{n-2}(h)=\frac{h^{n-1}}{(n-2) !} \int_{0}^{1} f^{(n-1)}(x+t h)(1-t)^{n-2} d t,
$$

where $p_{n-2} \in P_{n-2}$. Now let $g(u)=c u+d$ be an arbitrary linear function. Then for every $h$ with $x+h \in I$ we have

$$
\begin{aligned}
\int_{0}^{1} & \left(f_{+}^{(n-1)}(x+t h)-g(x+t h)-f_{+}^{(n-1)}(x)+g(x)\right) d \omega(t) \\
& =\int_{0}^{1}\left(f_{+}^{(n-1)}(x+t h)-f_{+}^{(n-1)}(x)-c t h\right) d \omega(t) \\
& =(n-1) n \int_{0}^{1}\left(f_{+}^{(n-1)}(x+t h)-f_{+}^{(n-1)}(x)-c t h\right)(1-t)^{n-2} d t \\
& =\frac{n !}{h^{n-1}}\left(f(x+h)-p_{n-2}(h)\right)-(n-1) n p_{1}(h) \\
& =\frac{n !}{h^{n-1}}\left(f(x+h)-p_{n}(h)\right)
\end{aligned}
$$

where $p_{1}$ is a linear function and $p_{n} \in P_{n}$. By assumption, $x$ is not a bilateral point of accumulation of the set $\left\{u ; f(u)=p_{n}(u-x)\right\}$ and hence $f(x+h)-p_{n}(h)$ does not change sign in a one-sided neighborhood of 0 . Therefore, $D^{\omega}\left(f_{+}^{(n-1)}-g\right)$ does not change sign at $x$ and this completes the proof of the theorem.

Corollary 14. If $f \in C[a, b]$ and $\{x ; f(x)=p(x)\}$ does not have any bilateral point of accumulation for every $p \in P_{n}$, then $f$ is $n-1$ times continuously differentiable on a subinterval of $[a, b]$.

ThEOREM 15. For every $f \in C[a, b]$ either there exists a polynomial $p$ such that $\{x ; f(x)=p(x)\}$ is infinite or there exists a function $g \in C^{\infty}$ such that $\{x ; f(x)=$ $g(x)\}$ is uncountable.

Proof. Let $f \in C[a, b]$ be given and suppose that $\{x ; f(x)=p(x)\}$ is finite for every polynomial $p$. Then, by Corollary 14 , for every $n>0$ and every subinterval $I \subset[a, b]$ there is a subinterval of $I$ on which $f$ is $n$ times continuously differentiable. We define a system of intervals $\left\{I_{i_{1}, \ldots, i_{n}} ; n \geq 0, i_{1}, \ldots, i_{n}=0,1\right\}$ as follows. Let $I_{\varnothing}$ be a closed subinterval of $[a, b]$ on which the oscillation of $f$ is less than 1. Suppose that the closed interval $I_{i_{1}, \ldots, i_{n}} \subset[a, b]$ has been defined. Then let $I_{i_{1}, \ldots, i_{n}, 0}$ and $I_{i_{1} \ldots, i_{n}, 1}$ be disjoint, closed subintervals of $I_{i_{1} \ldots, i_{n}}$ such that $f$ is $n+1$ times continuously differentiable on $\bigcup_{i=0}^{1} I_{i_{1}, \ldots, i_{n}, i}$ and the oscillation of $f^{(n+1)}$ on $I_{i_{1}, \ldots, i_{n}, i}$ is less than $2^{-(n+1)}(i=0,1)$. We put

$$
P=\bigcap_{n=0}^{\infty} \bigcup_{i_{1} \ldots, i_{n}=0,1} I_{i_{1} \ldots, i_{n}}
$$


It is easy to check that $f$ satisfies the conditions of Whitney's extension theorem on $P$ (see [9]). Thus there exists $g \in C^{\infty}$ such that $f(x)=g(x)$ for every $x \in P$, which proves the theorem.

Corollary 16. For every $f \in C[a, b]$ there exists $g \in C^{\infty}$ such that $\{x, f(x)=$ $g(x)\}$ is infinite.

4. Continuous functions defined on perfect sets. Let $P$ be a bounded, nowhere dense perfect set in $R$. We denote by $C(P)$ the Banach space of real valued, continuous functions defined on $P$ with the metric

$$
\rho(f, g)=\max \{|f(x)-g(x)| ; x \in P\} \quad(f, g \in C(P)) .
$$

First we show that in the space $C(P)$ the condition that the graph of $f$ intersects every line in finitely many (even at most two) points does not impose on $f$ any convexity or monotonicity property. In fact, we show that if $P$ is "small", then $f$ is nowhere monotonic and intersects every line in at most two points for all $f$ in a residual subset of $C(P)$.

LEMMA 17. There exists a nonempty perfect set $P$ such that whenever $f$ is a Lipschitz function of two variables defined on a rectangle $[a, b] \times[c, d]$, then

$$
A_{f}=\{f(x, y) ; x \in[a, b] \cap P, y \in[c, d] \cap P\}
$$

is nowhere dense.

Proof. We define $K_{\varnothing}=[0,1]$. If $i_{1}, \ldots, i_{n}$ is a finite $0-1$ sequence and the interval $K_{i_{1}, \ldots, i_{n}}$ is defined, then we select $K_{i_{1}, \ldots, i_{n}, 0}$ and $K_{i_{1}, \ldots, i_{n}, 1}$ as disjoint, closed subintervals of $K_{i_{1} \ldots, i_{n}}$ of length $5^{-n}$. In this way $K_{i_{1} \ldots, i_{n}}$ is defined by induction for every $n \geq 0$ and $i_{1}, \ldots, i_{n}=0,1$. Then

$$
P=\bigcap_{n=0}^{\infty} \bigcup_{i_{1}, \ldots, i_{n}=0,1} K_{i_{1}, \ldots i_{n}}
$$

is a nonempty perfect set which can be covered by $2^{n}$ intervals of length $5^{-n}$. Now let $f$ be defined on $[a, b] \times[c, d]$ and suppose that

$$
|f(x, y)-f(u, v)| \leq M(|x-u|+|y-v|) \quad((x, y),(u, v) \in[a, b] \times[c, d]) \text {. }
$$

It is easy to see that $A_{f}$ can be covered by $4^{n}$ intervals of length $2 M \cdot 5^{-n}$. Thus $\lambda\left(A_{f}\right)=0$ and, in particular, $A_{f}$ is nowhere dense.

THEOREM 18. Let $P$ be a nonempty, bounded perfect set satisfying the requirement of the previous lemma. Then the set of functions $f \in C(P)$ such that

(i) $f$ is not monotonic on any portion of $P$, and

(ii) the graph of $f$ does not contain three collinear points is a dense $G_{\delta}$ set in $C(P)$.

Proof. Let $Q$ denote the set of rationals. If $a, b \in Q, a<b$, and $(a, b) \cap P \neq \varnothing$, then it is easy to see that

$$
\mathscr{F}_{a, b}=\{f \in C(P) ; f \text { is monotonic on }[a, b] \cap P\}
$$

is a nowhere dense, closed subset of $C(P)$. 
Now let $a, b, c, d, e, f \in Q \backslash P, a<b<c<d<e<f$, and put

$$
\begin{array}{r}
\mathscr{G}=\mathscr{G}_{a, b, c, d, e, f}=\{g \in C(P) ; \text { there are points } x \in[a, b] \cap P, y \in[c, d] \cap P, \\
z \in[e, f] \cap P \text { such that }(x, g(x)),(y, g(y)), \\
(z, g(z)) \text { are collinear }\} .
\end{array}
$$

It is easy to see that $\mathscr{G}$ is closed in $C(P)$. Let $\mathscr{H}$ denote the set of functions $f \in C(P)$ satisfying (i) and (ii). Obviously, $C(P) \backslash \mathscr{H}$ is the union of the sets $\mathscr{F}_{a, b}$ and

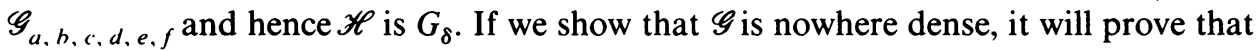
$\mathscr{H}$ is everywhere dense, since $C(P)$ is complete.

We have to show that for every $g_{0} \in C(P)$ and $\varepsilon>0$ there is $g \in C(P)$ such that $\rho\left(g, g_{0}\right)<\varepsilon$ and $g \notin \mathscr{G}$.

First we choose $g_{1} \in C(P)$ such that $\rho\left(g_{1}, g_{0}\right)<\varepsilon / 2$ and $g_{1}$ takes only finitely many values. Let

$$
g_{1}([a, b] \cap P)=\left\{a_{1}, \ldots, a_{k}\right\}, \quad g_{1}([c, d] \cap P)=\left\{b_{1}, \ldots, b_{n}\right\} .
$$

Let

$$
\begin{array}{r}
D=\{(u, v) ; \text { there are points } x \in[a, b] \cap P, y \in[c, d] \cap P \\
\text { such that } \left.\left(x, g_{1}(x)\right),\left(y, g_{1}(y)\right),(u, v) \text { are collinear }\right\} .
\end{array}
$$

Then $D$ is a closed subset of the plane.

We prove that for every fixed $u, D_{u}=\{v ;(u, v) \in D\}$ is nowhere dense. Indeed, if $v \in D_{u}$, then there are points $x \in[a, b] \cap P, y \in[c, d] \cap P$ and indices $i=$ $1, \ldots, k ; j=1, \ldots, n$ such that $\left(x, a_{i}\right),\left(y, b_{j}\right),(u, v)$ are collinear; that is

$$
v=\left(b_{j}-a_{i}\right) \frac{u-x}{y-x}+a_{i} \text {. }
$$

For every fixed $i, j$ the function

$$
f_{i, j}(x, y)=\left(b_{j}-a_{i}\right) \frac{u-x}{y-x}+a_{i}
$$

is continuously differentiable and hence Lipschitz 1 on the rectangle $[a, b] \times[c, d]$. Thus $A_{f_{t,}}$ is nowhere dense and so is

$$
D_{u} \subset \bigcup_{i=1}^{k} \bigcup_{j=1}^{n} A_{f_{i, j}} .
$$

Therefore for every $u \in[e, f] \cap P$ there is $\delta>0$ and $v \in \mathbf{R}$ such that $|g(z)-v|<$ $\varepsilon / 2$ and $(z, v) \notin D$ for every $|z-u|<\delta$. By the compactness of $[e, f] \cap P$ we can define a function $g$ on $[e, f] \cap P$ such that $\left|g(z)-g_{1}(z)\right|<\varepsilon / 2(z \in[e, f] \cap P)$ and

$$
\{(z, g(z)) ; z \in[e, f] \cap P\} \cap D=\varnothing .
$$

Let $g(z)=g_{1}(z)$ for $z \in P \backslash[e, f]$. Then $g$ satisfies our requirements and this completes the proof.

Next we remark that for every bounded $P$ of measure zero there exists $f \in C(P)$ such that $A_{g}=\{x \in P ; f(x)=g(x)\}$ is finite for every differentiable $g$. In fact, if $\lambda(P)=0$, then there is $f \in C(P)$ such that the derivative of $f$ with respect to $P$ is 
infinity at each $x \in P$. If $A_{g}$ were infinite for a differentiable $g$, then at a point $x$ of accumulation of $A_{g}$ we would have

$$
g^{\prime}(x)=\lim _{\substack{y \rightarrow x \\ y \in A_{g}}} \frac{g(y)-g(x)}{y-x}=\lim _{\substack{y \rightarrow x \\ y \in A_{g}}} \frac{f(y)-f(x)}{y-x}=\infty,
$$

which is impossible. Our next theorem shows that the situation is different if $\lambda(P)>0$.

TheOREM 19. Let $P \subset[a, b]$ be a perfect set of positive measure. Then for every $f \in C(P)$ there is $g \in C^{1}$ such that $\{x \in P ; f(x)=g(x)\}$ is uncountable.

LEMMA 20. Let $f$ be defined on the perfect set $S$ and suppose that $f$ is differentiable with respect to $S$ at every point of $S$. Then there is $g \in C^{1}$ such that $\{x \in S ; f(x)=$ $g(x)\}$ is uncountable.

Proof. We define

$$
\begin{array}{r}
S_{n, k}=\left\{x \in S ;\left|f(y)-f(x)-f^{\prime}(x)(y-x)\right| \leq(y-x) / n\right. \\
\text { for every } y \in S,|y-x| \leq 1 / k\} \\
\quad(n, k=1,2, \ldots) .
\end{array}
$$

Then $S_{n, k}$ is a Borel set for every $n, k=1,2, \ldots$ and $S_{n, 1} \subset S_{n, 2} \subset \cdots, S=\bigcup_{k=1}^{\infty} S_{n, k}$ $(n=1, \ldots)$. We prove that there is a sequence $\left\{k_{n}\right\}_{n=1}^{\infty}$ such that $\bigcap_{n=1}^{\infty} S_{n, k_{n}}$ is uncountable. There is a homeomorphism $\phi: S \rightarrow S^{\prime}$ such that $S^{\prime}=\phi(S)$ is perfect and $1<\lambda\left(S^{\prime}\right)<2$. Then $\phi\left(S_{n, k}\right)$ is measurable for every $n, k$ and $S^{\prime}=\bigcup_{k=1}^{\infty} \phi\left(S_{n, k}\right)$ $(n=1,2, \ldots)$. Let $k_{n}$ be chosen such that $\lambda\left(\phi\left(S_{n, k_{n}}\right)\right)>\lambda\left(S^{\prime}\right)-1 / 2^{n}(n=1,2, \ldots)$. Then $\lambda\left(\phi\left(\bigcap_{n=1}^{\infty} S_{n, k_{n}}\right)\right)=\lambda\left(\bigcap_{n=1}^{\infty} \phi\left(S_{n, k_{n}}\right)\right)>0$ and hence $\bigcap_{n=1}^{\infty} S_{n, k_{n}}$ is uncountable.

Let $S^{\prime \prime}$ be a perfect subset of $\bigcap_{n=1}^{\infty} S_{n, k_{n}}$. It is easy to check that $f$ satisfies the conditions of Whitney's extension theorem [9]. Thus there exists $g \in C^{\prime}$ with $f(x)=g(x)\left(x \in S^{\prime \prime}\right)$ which proves the lemma.

Proof of Theorem 19. Let $P \subset[a, b], \lambda(P)>0$ and let $f \in C(P)$. By Theorem 1 of [5] there exists a perfect subset $S \subset P$ such that $f$ is differentiable on $S$ (with respect to $S$ ). Thus Lemma 20 is applicable.

Corollary 21. For every $f \in C[a, b]$ there is $g \in C^{1}$ such that $\{x ; f(x)=g(x)\}$ is uncountable.

Our next theorem shows that Theorem 19 is the best possible.

THEOREM 22. For every $\varepsilon>0$ there exists a perfect set $P \subset[0,1]$ with $\lambda(P) \geq 1-\varepsilon$ and $f \in C(P)$ such that $\{x \in P ; f(x)=g(x)\}$ is finite for every $g$ which is twice differentiable on $[0,1]$.

Proof. We can suppose $0<\varepsilon<\frac{1}{2}$. We define the closed intervals $I_{i_{1}, \ldots, i_{n}}$ and the open intervals $J_{i_{1}, \ldots, i_{n}}$ for every finite $0-1$ sequence $i_{1}, \ldots, i_{n}$ as follows. We put $I_{\varnothing}=[0,1]$. Suppose that $I_{i_{1}, \ldots, i_{n}}$ has been defined and

$$
\left|I_{i_{1}, \ldots, i_{n}}\right|=2^{-n}\left(1-\sum_{k=0}^{n-1} 2^{k} \cdot \varepsilon \cdot 3^{-(k+1)}\right)
$$


Let $J_{i_{1} \ldots, i_{n}}$ denote the open interval of length $\varepsilon \cdot 3^{-n-1}$ and concentric with $I_{i_{1} \ldots, i_{n}}$. We denote by $I_{i_{1}, \ldots, i_{n}, 0}$ and $I_{i_{1} \ldots, i_{n}, 1}$ the components of $I_{i_{1}, \ldots, i_{n}} \backslash J_{i_{1}, \ldots, i_{n}}$. (This definition makes sense because

$$
\begin{aligned}
2^{-n}\left(1-\sum_{k=0}^{n-1} 2^{k} \varepsilon \cdot 3^{-(k+1)}\right) & >2^{-n}\left(1-\frac{\varepsilon}{3} \cdot \sum_{k=0}^{\infty}\left(\frac{2}{3}\right)^{k}\right) \\
& \left.=2^{-n}(1-\varepsilon)>\varepsilon \cdot 3^{-n-1} \cdot\right)
\end{aligned}
$$

Now we put

$$
P=\bigcap_{n=0}^{\infty} \bigcup_{i_{1}, \ldots, i_{n}=0,1} I_{i_{1} \ldots, i_{n}}
$$

Then $P$ is perfect and $\lambda(P)=1-\varepsilon$.

We define a function $h$ as follows: $h(x)=0$ if $x \in P$, let $h$ be continuous and linear in each half of the closure of $J_{i_{1}, \ldots, i_{n}}$ and let the value of $h$ at the midpoint of $J_{i_{1}}, \ldots i_{n}$ be $1 /(n+1)$ for every $n \geq 0, i_{1}, \ldots, i_{n}=0,1$. Then $h \in C[0,1]$. We define

$$
f(x)=\int_{0}^{x} h(t) d t \quad(x \in[0,1]) .
$$

Then $f \in C^{1}$ and $f^{\prime}(x)=0$ for every $x \in P$. Let $g$ be twice differentiable on $[0,1]$; we show that $A=\{x \in P ; f(x)=g(x)\}$ is finite. Suppose $A$ is infinite. Then there is a convergent sequence $x_{k} \rightarrow x_{0}, x_{k} \neq x_{0}, x_{k} \in A(k=1,2, \ldots)$. Now, $f^{\prime}\left(x_{0}\right)=0$ so $g^{\prime}\left(x_{0}\right)=0$ and, by L'Hôpital's rule,

$$
\lim _{x \rightarrow x_{0}} \frac{g(x)-g\left(x_{0}\right)}{\left(x-x_{0}\right)^{2}}=\lim _{x \rightarrow x_{0}} \frac{g^{\prime}(x)}{2\left(x-x_{0}\right)}=\frac{1}{2} g^{\prime \prime}\left(x_{0}\right)
$$

Since $x_{k} \in A$, this implies

$$
\lim _{k \rightarrow \infty} \frac{f\left(x_{k}\right)-f\left(x_{0}\right)}{\left(x_{k}-x_{0}\right)^{2}}=\frac{1}{2} g^{\prime \prime}\left(x_{0}\right) .
$$

On the other hand, for every $k \geq 0$ there are $n \geq 0$ and $i_{1}, \ldots, i_{n}=0,1$ such that $x_{0}, x_{k} \in I_{i_{1} \ldots i_{n}}$. Suppose that $n$ is the largest integer for which there are $i_{1}, \ldots, i_{n}$ with this property. Since $x_{0}, x_{k} \in P$, this implies that $x_{0} \in I_{i_{1}, \ldots, i_{n}, 0}, x_{k} \in I_{i_{1}, \ldots, i_{n}, 1}$, or the other way around. Therefore

$$
\left|f\left(x_{k}\right)-f\left(x_{0}\right)\right|=\left|\int_{x_{0}}^{x_{k}} h(t) d t\right| \geq \int_{J_{i_{1}} \ldots, i_{n}} h(t) d t=\frac{1}{2} \cdot \frac{1}{n+1} \cdot \varepsilon \cdot 3^{-n-1}
$$

and

$$
\left|x_{k}-x_{0}\right| \leq\left|I_{i_{1} \ldots, i_{n}}\right| \leq 2^{-n}
$$

Consequently,

$$
\lim _{k \rightarrow \infty}\left|\frac{f\left(x_{k}\right)-f\left(x_{0}\right)}{\left(x_{k}-x_{0}\right)^{2}}\right| \geq \lim _{n \rightarrow \infty} 4^{n} \cdot \frac{1}{2} \cdot \frac{1}{n+1} \cdot \varepsilon \cdot 3^{-n-1}=\infty,
$$

a contradiction. Thus $A$ is finite and the proof is complete. 
5. Remarks on a combinatorial problem. By a theorem of Filipczak [3], for every $f \in C[a, b]$ there is a perfect set $P \subset[a, b]$ on which $f$ is monotone. This result can be regarded as the continuous variant of the following combinatorial theorem. If $E$ is an infinite subset of the real line and $f: E \rightarrow R$ is arbitrary, then there is an infinite subset $H \subset E$ such that $f$ is monotonic on $H$. This is an immediate consequence of Ramsey's theorem [8, p. 33]. In fact, if $G$ denotes the graph $\{(x, y) ; x, y \in E$, $x \neq y,(f(y)-f(x)) /(y-x)>0\}$ then, by Ramsey's theorem, there is an infinite subset $H \subset E$ such that either $G_{1}=\{(x, y) ; x, y \in H, x \neq y\} \subset G$ or $G_{1} \cap G=\varnothing$ and then $f$ is obviously monotonic on $H$. This theorem easily extends to $n$-convexity. Indeed, since Ramsey's theorem is valid for hypergraphs as well, we get an infinite subset $H \subset E$ on which the $n$th divided differences of $f$ are of the same sign, that is on which $f$ is either $n$-convex or $n$-concave. Thus the question arises whether the continuous variant of this theorem is valid. In other words, we can formulate the following problem.

Let $f \in C[a, b]$ be arbitrary and let $n$ be a nonnegative integer. Does there exist a nonempty perfect set $P \subset[a, b]$ on which $f$ is either $n$-convex or $n$-concave?

We remark that for continuous functions defined on perfect sets, the answer is negative. In fact, let $f$ be a strictly increasing, continuous function defined on the perfect set $P$ such that the derivative of $f$ with respect to $P$ is zero at every $x \in P$. It is easy to check that if $f$ is convex or concave on a subset $H \subset P$, then $H$ does not contain two different points of accumulation. Thus $H$ must be countable and hence $f$ is not 2-convex or 2-concave on any perfect subset of $P$.

\section{REFERENCES}

1. H. Cartan, Collected works, Springer, Berlin and New York, 1979.

2. E. Čech, Sur les fonctions continues qui prennent chaque leur valeur un nombre fini de fois, Fund. Math. 17 (1931), 32-39.

3. F. Filipczak, Sur les fonctions continues relativement monotones, Fund. Math. 58 (1966), 75-87.

4. K. M. Garg, On level sets of a continuous nowhere monotone function, Fund. Math. 52 (1963), 59-68.

5. M. Laczkovich, Differentiable restrictions of continuous functions, Acta Math. Acad. Sci. Hungar. (to appear).

6. K. Padmavally, On the roots of equation $f(x)=\xi$ where $f(x)$ is real and continuous in $(a, b)$ but monotonic in no subinterval of $(a, b)$, Proc. Amer. Math. Soc. 4 (1953), 839-841.

7. S. M. Ulam, A collection of mathematical problems, Interscience, New York, 1960.

8. N. H. Williams, Combinatorial set theory, North-Holland, Amsterdam, 1977.

9. H. Whitney, Analytic extensions of differentiable functions defined in closed sets, Trans. Amer. Math. Soc. 36 (1934), 63-89.

10. Z. Zahorski, Sur l'ensemble des points singuliers d'une fonction d'une variable réele admittant les dérivés de tous les ordres, Fund. Math. 34 (1947), 183-245.

Department of Mathematics, California Polytechnic State University, San luis Obispo, CALIFORNIA 93407

Department of Mathematics, University of California, Santa Barbara, California 93106

DePartment of ANALYSIS, EOTVOS LORÁND UNIVERSity, 1088 BudAPEST, MÚzeum KRT. $6-8$, HUNGARY

MFFUK Sokolovská 83, 18600 Prague 8, Czechoslovakia 